\title{
HCN Observations of Submillimeter Galaxies and QSOs at High Redshift
}

\author{
Yu Gao ${ }^{1,2}$, C.L. Carilli ${ }^{2}$, P.M. Solomon ${ }^{3}$ \& P.A. Vanden Bout ${ }^{4}$ \\ ${ }^{1}$ Purple Mountain Observatory, 2 West Beijing Road, Nanjing 210008; and National \\ Astronomy Observatory, Chinese Academy of Sciences, Beijing, P.R. China \\ yugao@pmo.ac.cn,ygao@nrao.edu \\ ${ }^{2}$ National Radio Astronomy Observatory, Socorro, NM 87801, USA \\ ${ }^{3}$ Department of Physics \& Astronomy, SUNY at Stony Brook, Stony Brook, NY 11794, USA \\ ${ }^{4}$ National Radio Astronomy Observatory, Charlottesville, VA 22903, USA
}

We have conducted sensitive $\mathrm{HCN}(1-0)$ line observations of four high redshift submillimeter (sub-mm) galaxies and QSOs with the VLA. Although none is significantly detected in either line or continuum emission, thanks to the gravitational lens, sub-mm galaxy SMM J16359+6612 is marginally (at the 3-4 $\sigma$ level) detected once the three lensed components are stacked up. The HCN source appears to be double with a separation of $\sim 1.5^{\prime \prime}$, consistent with what has been implied from other observations. This first possible $\mathrm{HCN}$ detection of a submm galaxy, and three stringent $\mathrm{HCN}$ upper limits, combined with previous $\mathrm{HCN}$ detections and upper limits strongly constrain the $\mathrm{HCN} / \mathrm{CO}$ ratios at high-z to be comparable to that of local ULIRGs.

A total of 16 hours VLA time were obtained for SMM J16359. The larger image shows $\mathrm{HCN}$ emission from SMM J16359, visible at the position of the CO component B (mid-cross). Note the position of $\mathrm{CO}$ component A also shows possible $\mathrm{HCN}(\sim 3 \sigma)$ detection at exactly the position of the $\mathrm{CO}$ Component A centroid (upperleft cross). The inset image was constructed by overlaying the positions of the three CO components and taking a weighted average. The centroid of CO component $\mathrm{B}$ is at position J2000 RA: 1635 54.1 DEC: 66 12 23.8, directly between the two possible HCN components.

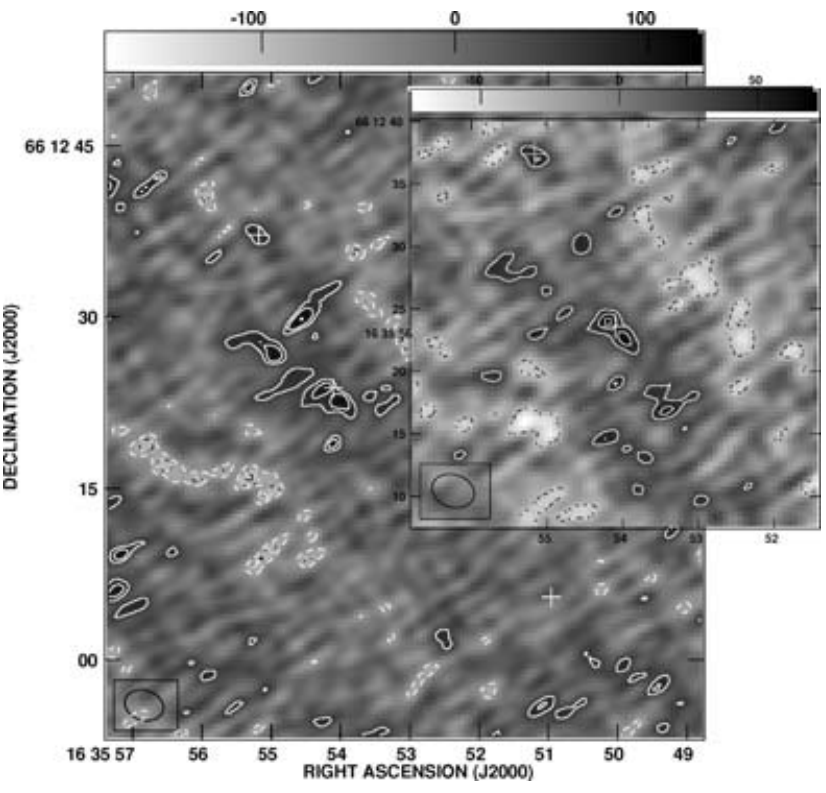
Although unresolved in $\mathrm{CO}$, the elogation of CO morphology and the separation of the blueshifted and redshifted velocity components are all consistent with our observed HCN morphology.

China NSF \& Chinese Academy of Sciences (YG) and Max-Planck Society (CLC) are thanked for supports. 